\title{
Vector competence of Aedes albopictus for the Indian Ocean lineage (IOL) chikungunya viruses of the 2007 and 2017 outbreaks in Italy: a comparison between strains with and without the E1:A226V mutation
}

\author{
Claudia Fortuna ${ }^{1,2}$, Luciano Toma ${ }^{2,3}$, Maria Elena Remoli ${ }^{1}$, Antonello Amendola ${ }^{1}$, Francesco Severini ${ }^{3}$, Daniela Boccolini ${ }^{3}$, Roberto \\ Romi ${ }^{3}$, Giulietta Venturi' ${ }^{1}$, Giovanni Rezza ${ }^{1}$, Marco Di Luca ${ }^{3}$ \\ 1. Istituto Superiore di Sanità, Department of Infectious Diseases, National Reference Laboratory for Arboviruses, Rome, Italy \\ 2. These authors contributed equally to this article and share first authorship \\ 3. Istituto Superiore di Sanità, Department of Infectious Diseases, Unit of Vector-borne Diseases, Rome, Italy
}

Correspondence: Claudia Fortuna (claudia.fortuna@iss.it)

Citation style for this article:

Fortuna Claudia, Toma Luciano, Remoli Maria Elena, Amendola Antonello, Severini Francesco, Boccolini Daniela, Romi Roberto, Venturi Giulietta, Rezza Giovanni, Di Luca Marco. Vector competence of Aedes albopictus for the Indian Ocean lineage (IOL) chikungunya viruses of the 2007 and 2017 outbreaks in Italy: a comparison between strains with and without the E1:A226V mutation. Euro Surveill. 2018;23(22):pii=1800246. https://doi.0rg/10.2807/1560-7917.ES.2018.23.22.1800246

We compared the vector competence of an Italian population of Aedes albopictus for two strains of chikungunya virus (CHIKV), with and without E1:A226V mutation, responsible for outbreaks in 2007 in the Emilia Romagna region and 2017 in the Lazio and Calabria regions, respectively. Ae. albopictus showed similar vector competence for both viral strains indicating that E1:A226V mutation is not exclusively responsible for ability of CHIKV to replicate well in this mosquito species.

Over the last decade, Ae. albopictus has been responsible for two large chikungunya virus (CHIKV) outbreaks in Italy, by two different East-Central-South African (ECSA) Indian Ocean lineage (IOL) strains of the virus: the strain with the Ae. albopictus-adaptive E1:A226V mutation (2007 outbreak in Emilia Romagna region) and without this mutation (2017 outbreak in Lazio and Calabria regions) [1-3]. The aim of our study was to experimentally evaluate and compare the vector competence (VC) of Ae. albopictus mosquitoes for both strains of CHIKV.

\section{Experimental infection of mosquitoes}

In the late summer of 2015 , a colony of Italian Ae. Albopictus was collected in Scalea town, Calabria region and established in our laboratory [4]. The longestablished colony was subsequently used in the parallel experimental infection with two strains of CHIKV ECSA IOL with and without the E1:A226V mutation (Accession number LT908478 and LS453285, respectively). The CHIKV strains were isolated from biological samples of two patients infected during the Italian outbreaks in 2007 and 2017 and used in the current study after amplification in Vero cells. Oral infection was performed in a BSL3 laboratory where 5 to 8 day-old mosquito females were exposed to an infectious blood meal for 60 min via a membrane feeder apparatus. The apparatus contained the virus stock diluted 1:3 in rabbit blood with a final virus concentration of 6.58 $\log _{10}$ plaque forming unit (PFU)/mL for CHIKV carrying the mutation (CHIKVE1:A226V) and $6.99 \log _{10} \mathrm{PFU} / \mathrm{mL}$ for the virus lacking the mutation (CHIKVE1).

After the blood meal, fully engorged females were transferred into dedicated cages and maintained on a $10 \%$ sucrose solution in a climatic chamber $\left(T=26 \pm 1{ }^{\circ} \mathrm{C}\right.$, $70 \% \mathrm{RH}, 14 / 10 \mathrm{~h}$ light/dark cycle) and monitored for 20 days.

A sample of mosquitoes (10 specimens) were individually processed for each viral strain at 0, 3, 14 and 20 days post-exposure (dpe). The viral presence in body, legs plus wings and saliva of the mosquitoes was analysed. CHIKV titre was evaluated with quantitative Real Time PCR using specific primers and probe [1]. Crossing point values were compared with a standard curve obtained from 10 -fold serial dilutions of virus stock whose concentration was estimated by titration on Vero cells and expressed as $\log _{10} P F U / m L$.

Infection rate (IR) was calculated as the number of positive bodies/total fed females, while the dissemination rate (DR) and transmission rate (TR) were calculated as the number of positive legs plus wings/ positive bodies and the number of positive saliva/ positive bodies, respectively, as previously described [5]. To consider the effective transmission ability of a population of experimentally infected Ae. albopictus, the transmission efficiency (TE) was also calculated as 
Mean titres in bodies, legs plus wings and saliva in Italian Aedes albopictus experimentally infected with CHIKVE1:A226V (A) and CHIKVE1 (B) at different days post-exposure $(\mathrm{n}=10)$

A. CHIKVE1:A226V

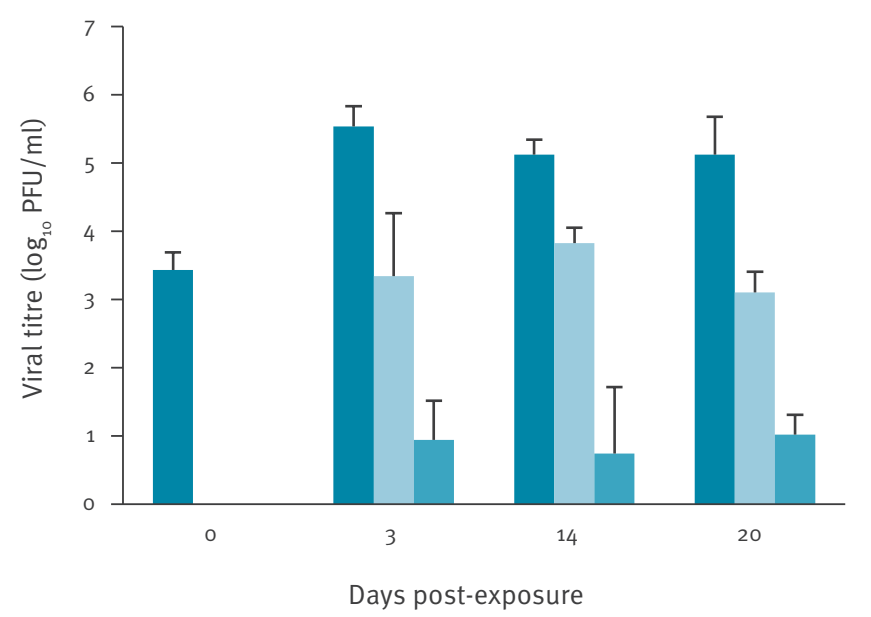

B. CHIKVE1

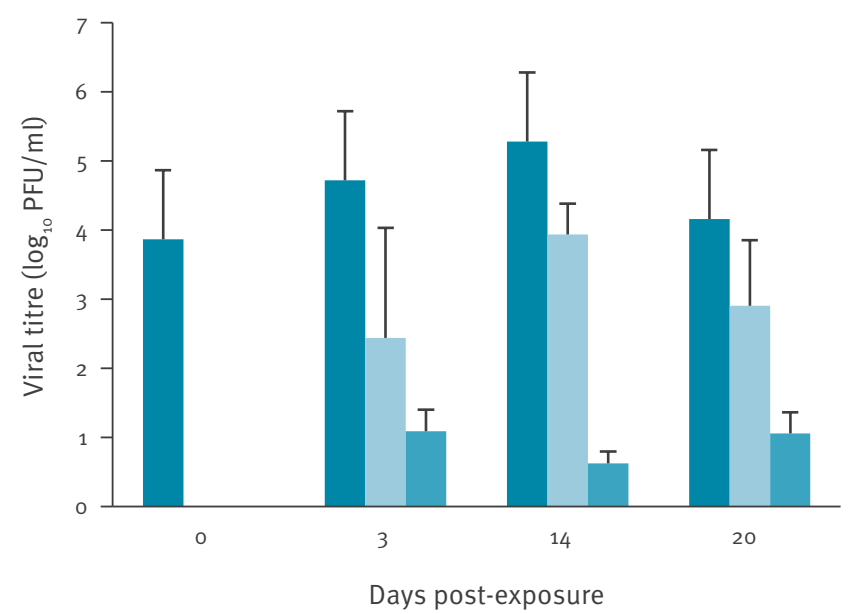

Body Legs and wings $\square$ Saliva

CHIKVE1:A226V: chikungunya virus strain with E1:A226V mutation.

CHIKVE1: chikungunya virus strain without E1:A226V mutation.

the number of positive saliva/total fed females, as previously reported [6].

Data were analysed in the GraphPad Prism statistical PC programme (GraphPad Software, San Diego, California, United States) using unpaired t-test. A level of $p<0.05$ was considered statistically significant.

\section{Vector competence analysis}

The viral titres and the values of IR, DR, and TR, at all collection points, are shown in Figure 1 and Figure 2. A sample of engorged mosquitoes was analysed immediately after the infectious blood meal (day o) to verify the ingestion of infectious viral particles. All tested specimens resulted positive with a viral titre of $3.43 \pm 0.26 \log _{10} \mathrm{PFU} / \mathrm{mL}$ for CHIKVE1:A226V and $3.86 \pm 0.13 \log _{10} \mathrm{PFU} / \mathrm{mL}$ for CHIKVE1 (Figure 1). The titre increased progressively in the bodies collected at 3 and 14 dpe for both viral strains. Differences in IR values between the two viral strains were observed only at 3 dpe, but these were not statistically significant ( $>$ >0.05). IR percentages became progressively more similar at two subsequent collection times (Figure 2A). The extrinsic incubation period (EIP) was very short for both viral strains: viral presence was detected in the saliva starting from 3 dpe for CHIKVE1:A226V and CHIKVE1, with a mean viral titre of $0.94 \pm 0.57 \log _{10} \mathrm{PFU} /$ $\mathrm{mL}$ and $1.08 \pm 0.31 \log _{10} \mathrm{PFU} / \mathrm{mL}$, respectively. The highest viral titre in legs plus wings was detected at 14 dpe for both strains, with a titre of $3.82 \pm 0.22 \log _{10}$ PFU/ $\mathrm{mL}$ (CHIKVE1:A226V) and 3.93 $0.45 \log _{10} \mathrm{PFU} / \mathrm{mL}$
(CHIKVE1) (Figure 1). The DR values were $100 \%$ for CHIKVE1:A226V at each collection time, whereas they ranged from $75 \%$ to $100 \%$ for CHIKVE1 (Figure $2 \mathrm{~B}$ ).

In the saliva samples of both strains, the viral genome was detected already 3 dpe and up to $20 \mathrm{dpe}$, demonstrating the capacity of Ae. albopictus to maintain active viral replication for a long time (Figure 1). A constant value of the viral titre was detected from saliva at all collection times. For both CHIKV strains, TR values ranged between $50 \%$ and $86 \%$ for CHIKVE1:A226V and between $75 \%$ and $80 \%$ for CHIKVE1 (Figure $2 \mathrm{C}$ ), with differences that were not statistically significant ( $p>0.05$ ) between the two viral strains, in all collection times.

In order to assess the overall risk of transmission of the two CHIKV strains by Ae. albopictus, the TE was calculated for the whole time interval in which mosquitoes showed the virus in the saliva (i.e. from 3 to $20 \mathrm{dpe}$ ), regardless of the different collection times. Comparable rates were detected: $41 \%(12 / 29)$ of $A e$. albopictus infected with CHIKVE1:A226V and $42 \%$ $(11 / 26)$ with CHIKVE1, were able to secrete virus in the saliva.

\section{Discussion and conclusion}

The recent emergence and re-emergence of arboviruses such as Zika, yellow fever, and chikungunya, and their recent spread, have generated global concern. Arboviral infections transmitted by mosquitoes 
Infection(A), dissemination (B), transmission (C) rates of Italian Aedes albopictus experimentally infected with CHIKVE1:A226V and CHIKVE1 by days post-exposure

A. Infection

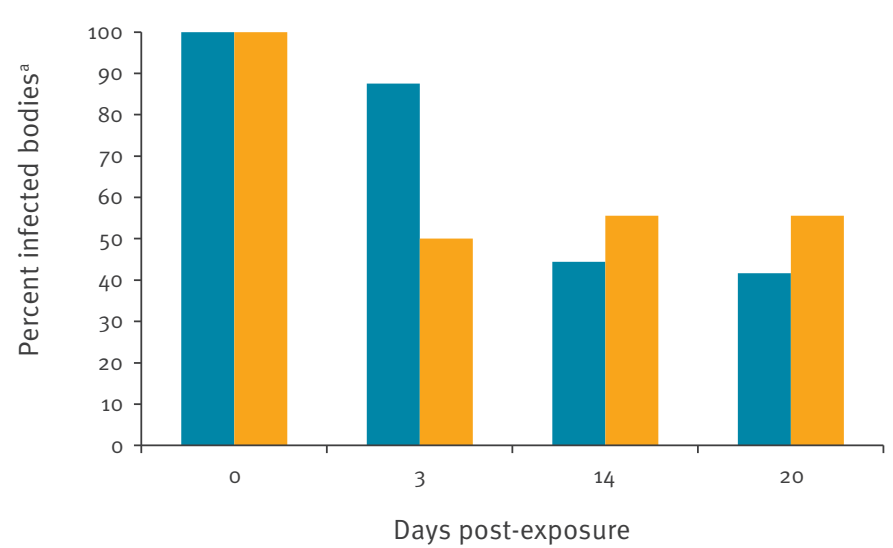

C. Transmission

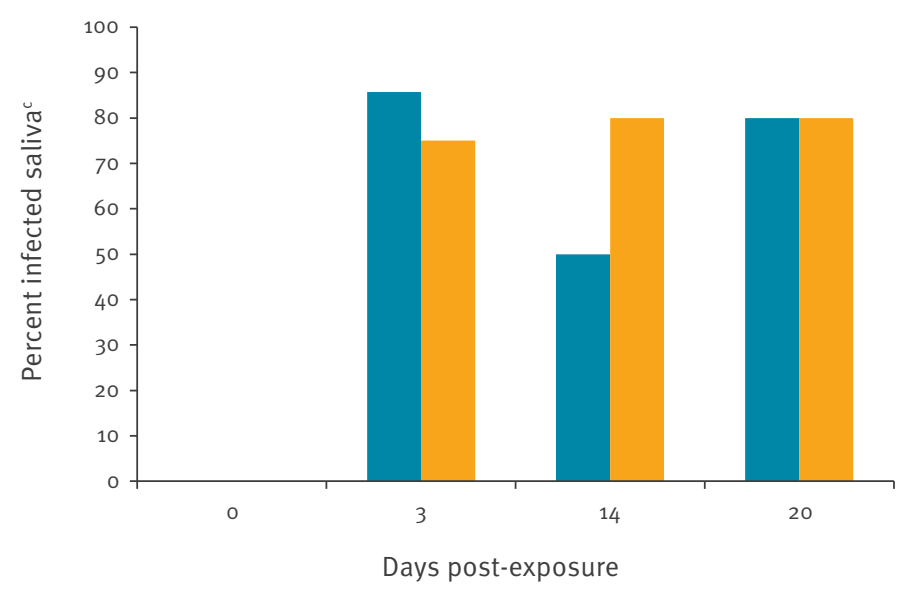

CHIKVE1:A226V: chikungunya virus strain with E1:A226V mutation. CHIKVE1: chikungunya virus strain without E1:A226V mutation.

${ }^{a}$ Number of positive bodies/number of engorged females.

${ }^{b}$ Number of positive legs plus wings/number of positive bodies.

'Number of positive saliva/number of positive bodies.

are often difficult to diagnose as symptoms are similar to other infectious diseases and can cause clinical complications that can be severe [7]. In August 2017, locally-acquired CHIKV infections occurred in SouthEast France and in Central and South Italy, highlighting the vulnerability of Europe to the transmission of tropical arboviruses [2,8].

Both chikungunya outbreaks that occurred in Italy during 2007 and 2017 originated from imported human cases of infection; the first characterised by the E1:A226V mutation and the second by its absence. The two regions affected by the outbreaks had similar environmental and climatic characteristics at the time of the outbreaks: they both consisted of small villages

\section{B. Dissemination}

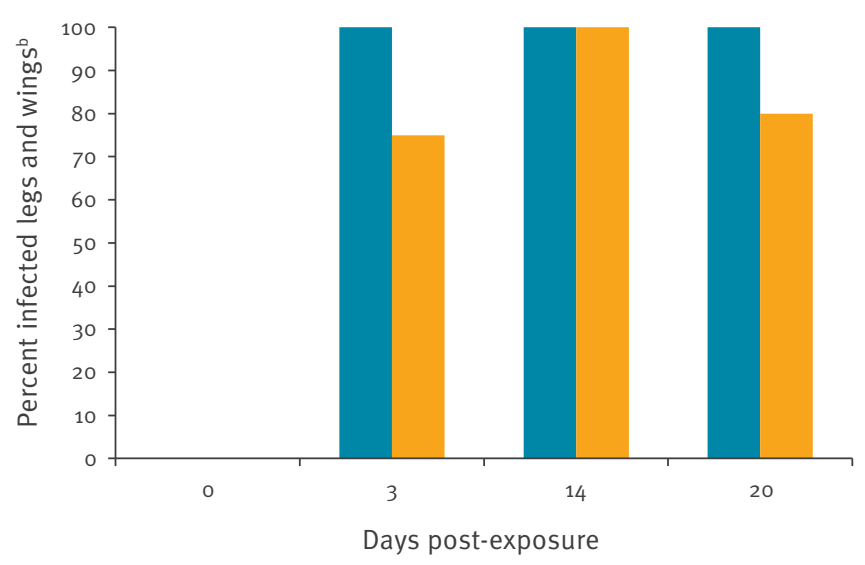

CHIKVE1:A226V

CHIKVE1

with detached-housing and gardens, summers were warm with temperatures above the seasonal average and there was a local high abundance of Ae. albopictus. Entomological investigations carried out during the outbreaks, highlighted the presence of numerous peridomestic or 'man-made' breeding sites within shady and constantly irrigated private gardens, sites suitable for the development of Ae. albopictus larvae [2]. These, along with a delay in clinical diagnosis of chikungunya infection at the time of the outbreaks, favoured the spread of both viral epidemics.

The first outbreak of chikungunya was caused by a strain carrying the $\mathrm{E}_{1} \mathrm{~A}$ A226V mutation, which is believed to improve the capacity of the virus to replicate in $A e$. 
albopictus [9]. CHIKV strain isolated during the Italian outbreak in 2017 did not carry this mutation. It was for this reason, that we focused our study on the comparison of the VC of Ae. albopictus for the two Italian CHIKV strains, with and without the E1:A226V mutation.

Our results show a comparable VC of Ae. albopictus for both CHIKV strains, with similar IR, DR, TR, and TE: no statistically significant difference was observed between the two strains. These findings, along with the dynamics and the extent of the outbreaks occurring in 2017, highlight that the two viral strains analysed here both infect, disseminate and are transmitted similarly to each other supporting the data previously published [10]. In addition, the viral isolation from mosquito pools collected during the 2017 Italian outbreaks, suggest a high prevalence of CHIKV among circulating mosquito populations. In fact, viral isolation from field collected mosquitoes is usually relatively rare $[2,3]$.

The detection of viral particles of both CHIKV strains in the saliva of mosquitoes, starting from $3 \mathrm{dpe}$, highlighted a very short EIP. In previous investigations, analysis to determine presence of virus in mosquito saliva was limited until 14 dpe [11-13], whereas in our study detection was carried out for a further 6 days. This demonstrated the ability of Ae. albopictus to permit CHIKV to replicate for a longer time, prolonging the potential risk of viral transmission during an outbreak. Although the acquired mutation E1:A226V in IOL lineage allowed an increased efficacy of replication and dissemination of CHIKV, and a better viral adaptation in Ae. albopictus mosquitoes $[9,14,15]$, our data demonstrate that this mutation cannot be considered exclusively responsible for the virus's ability to better replicate in Ae. albopictus. Vega-Rua et al. also showed the high VC of Ae. albopictus for the CHIKV without mutation supporting our findings [16]. Another substitution at position E2-211, with a threonine instead of an isoleucine, may increase the infectivity of CHIKV for Ae. albopictus when expressed together with the E1:A226V mutation [17]. Since the CHIKV responsible for the 2017 Italian outbreak does not carry the E1:A226V mutation, it is plausible that there may be more mutations responsible for the high infectivity in Ae. albopictus of this strain. Further studies of genomic analysis should be performed, in order to identify other possible mutations that allow CHIKVE1 to infect this vector mosquito species.

\section{Conflict of interest}

None declared.

\section{Authors' contributions}

CF, MDL, MER, GV conceived study; MDL, DB, LT, FS, CF, MER were responsible for carrying out the experimental infections; CF, MER, AA were responsible for carrying out the sample analysis; CF, MER, GV carried out the data analysis and
CF, MDL, GR, RR drafted the manuscript. All authors read and approved the final manuscript.

\section{References}

1. Rezza G, Nicoletti L, Angelini R, Romi R, Finarelli AC, Panning $M$, et al. CHIKV study group. Infection with chikungunya virus in Italy: an outbreak in a temperate region. Lancet. 2007;370(9602):1840-6. https://doi.org/10.1016/S01406736(07)61779-6 PMID: 18061059

2. Venturi G, Di Luca M, Fortuna C, Remoli ME, Riccardo F, Severini F, et al. Detection of a chikungunya outbreak in Central Italy, August to September 2017. Euro Surveill. 2017;22(39). https://doi.org/10.2807/1560-7917.

ES.2017.22.39.17-00646 PMID: 29019306

3. European Centre for Disease Prevention and Control (ECDC). Clusters of autochthonous chikungunya cases in Italy. Stockholm: ECDC; 9 October 2017. Available from: https://ecdc. europa.eu/sites/portal/files/documents/RRA-chikungunyaItaly-update-9-Oct-2017.pdf

4. Di Luca M, Severini F, Toma L, Boccolini D, Romi R, Remoli ME, et al. Experimental studies of susceptibility of Italian Aedes albopictus to Zika virus. Euro Surveill. 2016;21(18):30223. https://doi.org/10.2807/1560-7917.ES.2016.21.18.30223 PMID: 27171034

5. Fortuna C, Remoli ME, Di Luca M, Severini F, Toma L, Benedetti $E$, et al. Experimental studies on comparison of the vector competence of four Italian Culex pipiens populations for West Nile virus. Parasit Vectors. 2015;8(1):463. https://doi. org/10.1186/s13071-015-1067-z PMID: 26383834

6. Amraoui F, Vazeille M, Failloux AB. French Aedes albopictus are able to transmit yellow fever virus. Euro Surveill. 2016;21(39):30361. https://doi.org/10.2807/1560-7917. ES.2016.21.39.30361 PMID: 27719755

7. Paixão ES, Teixeira MG, Rodrigues LC. Zika, chikungunya and dengue: the causes and threats of new and re-emerging arboviral diseases. BMJ Glob Health. 2018;3(Suppl 1):e000530. PMID: 29435366

8. Calba C, Guerbois-Galla M, Franke F, Jeannin C, Auzet-Caillaud M, Grard G, et al. Preliminary report of an autochthonous chikungunya outbreak in France, July to September 2017. Euro Surveill. 2017;22(39). https://doi.org/10.2807/1560-7917. ES.2017.22.39.17-00647 PMID: 29019313

9. Tsetsarkin KA, Vanlandingham DL, McGee CE, Higgs S. A single mutation in chikungunya virus affects vector specificity and epidemic potential. PLoS Pathog. 2007;3(12):e201. https://doi. org/10.1371/journal.ppat.0030201 PMID: 18069894

10. Severini F, Boccolini D, Fortuna C, Di Luca M, Toma L, Amendola $A$, et al. Vector competence of Italian Aedes albopictus populations for the chikungunya virus (E1-226V). PLoS Negl Trop Dis. 2018;12(4):eooo6435. https://doi.org/10.1371/ journal.pntd.0006435 PMID: 29672511

11. Vega-Rúa A, Zouache K, Girod R, Failloux AB, Lourenço-deOliveira R. High level of vector competence of Aedes aegypti and Aedes albopictus from ten American countries as a crucial factor in the spread of Chikungunya virus. J Virol. 2014;88(11):6294-306. https://doi.org/10.1128/JVI.00370-14 PMID: 24672026

12. Ngoagouni $C$, Kamgang B, Kazanji M, Paupy C, Nakouné E. Potential of Aedes aegypti and Aedes albopictus populations in the Central African Republic to transmit enzootic chikungunya virus strains. Parasit Vectors. 2017;10(1):164. https://doi. org/10.1186/s13071-017-2101-0 PMID: 28347325

13. van den Hurk AF, Hall-Mendelin S, Pyke AT, Smith GA, Mackenzie JS. Vector competence of Australian mosquitoes for chikungunya virus. Vector Borne Zoonotic Dis. 2010;10(5):48995. https://doi.org/10.1089/vbz.2009.0106 PMID: 19877822

14. Arankalle VA, Shrivastava S, Cherian S, Gunjikar RS, Walimbe AM, Jadhav SM, et al. Genetic divergence of Chikungunya viruses in India (1963-2006) with special reference to the 2005 2006 explosive epidemic. J Gen Virol. 2007;88(Pt 7):1967-76. https://doi.org/10.1099/vir.0.82714-0 PMID: 17554030

15. Vazeille M, Moutailler S, Coudrier D, Rousseaux C, Khun $H$, Huerre $M$, et al. Two Chikungunya isolates from the outbreak of La Reunion (Indian Ocean) exhibit different patterns of infection in the mosquito, Aedes albopictus. PLoS One. 2007;2(11):e1168. https://doi.org/10.1371/journal. pone.0001168 PMID: 18000540

16. Vega-Rua A, Zouache K, Caro V, Diancourt L, Delaunay P, Grandadam M, et al. High efficiency of temperate Aedes albopictus to transmit chikungunya and dengue viruses in the Southeast of France. PLoS One. 2013;8(3):e59716. https://doi. org/10.1371/journal.pone.0059716 PMID: 23527259 
17. Tsetsarkin KA, McGee CE, Volk SM, Vanlandingham DL, Weaver SC, Higgs S. Epistatic roles of E2 glycoprotein mutations in adaption of chikungunya virus to Aedes albopictus and Ae. aegypti mosquitoes. PLoS One. 2009;4(8):e6835. https://doi. org/10.1371/journal.pone.0006835 PMID: 19718263

\section{License and copyright}

This is an open-access article distributed under the terms of the Creative Commons Attribution (CC BY 4.0) Licence. You may share and adapt the material, but must give appropriate credit to the source, provide a link to the licence, and indicate if changes were made.

This article is copyright of the authors, 2018. 